University of Nebraska - Lincoln

DigitalCommons@University of Nebraska - Lincoln

\title{
Clark's Nutcrackers (Nucifraga columbiana) and the Effects of Goal-Landmark Distance on Overshadowing
}

Aleida J. Goodyear

University of Nebraska - Lincoln, ajgoodyear@hotmail.com

Alan Kamil

University of Nebraska - Lincoln, akamil1@unl.edu

Follow this and additional works at: https://digitalcommons.unl.edu/bioscibehavior

Part of the Behavior and Ethology Commons

Goodyear, Aleida J. and Kamil, Alan, "Clark's Nutcrackers (Nucifraga columbiana) and the Effects of GoalLandmark Distance on Overshadowing" (2004). Papers in Behavior and Biological Sciences. 16.

https://digitalcommons.unl.edu/bioscibehavior/16

This Article is brought to you for free and open access by the Papers in the Biological Sciences at DigitalCommons@University of Nebraska - Lincoln. It has been accepted for inclusion in Papers in Behavior and Biological Sciences by an authorized administrator of DigitalCommons@University of Nebraska - Lincoln. 
Published in Journal of Comparative Psychology 118:3 (2004), pp. 258-264; doi: 10.1037/0735-7036.118.3.258 Copyright (C) 2004 American Psychological Association. Used by permission. "This article may not exactly replicate the final version published in the APA journal. It is not the copy of record."

This experiment forms part of the dissertation of Aleida J. Goodyear. Some of the data were presented at the Animal Behavior Society meeting in July 2001, Corvallis, Oregon. We thank Debbie Kelly for comments on drafts of this article. Max Post van der Berg, Sarah Kippenbrock, Sara Jacobitz, and Cheri Westphal all provided assistance in conducting the experiment. This research was supported by National Institutes of Health Grant NH-61810 and by the University of Nebraska Initiative for Ecological and Evolutionary Analysis.

\title{
Clark's Nutcrackers (Nucifraga columbiana) and the Effects of Goal-Landmark Distance on Overshadowing
}

\author{
Aleida J. Goodyear \& Alan C. Kamil \\ School of Biological Sciences, University of Nebraska-Lincoln \\ Corresponding author - Aleida J. Goodyear or Alan C. Kamil, School of Biological Sciences, \\ University of Nebraska-Lincoln, Lincoln, NE 68588-0118; email ajgoodyear@hotmail.com or akamil@unlserve.unl.edu
}

\begin{abstract}
Three groups of Clark's nutcrackers (Nucifraga columbiana) were trained to find a goal location defined by an array of 4 landmarks that varied in goal-landmark distance. The arrays for each group differed in the distance of the closest landmark and contained goal-landmark distances that were common across groups, allowing for the examination of the effects of both relative and absolute goal-landmark distance on encoding of a landmark array. All 3 groups readily learned the task and were subsequently tested in probe tests with only single landmarks from the array available. Search error in tests with single landmarks was compared both within and across groups. Results demonstrated that both relative and absolute goal-landmark distances are important in spatial search.
\end{abstract}

Although it is well-known that animals frequently use landmarks during spatial search (see reviews by Cheng \& Spetch, 1998; Kamil \& Cheng, 2001), the nature of the encoding of landmark arrays is not well understood (Cheng \& Spetch, 1998). Although many species use landmarks, the landmarks within an array are often weighted unequally (Bennett, 1993; Basil, 1993; Cheng, 1992; Collett, Cartwright, \& Smith, 1986), frequently as a function of the distance between goal and landmarks. For example, pigeons (Cheng, 1992), gerbils (Collett et al., 1986), and bees (Cheng, Collett, Pickhard, \& Wehner, 1987) have been shown to only follow a shift of the closest landmark in an array. European jays (Bennett, 1993) and Clark's nutcrackers (Basil, 1993) exhibit more accurate search when more proximate landmarks in an array are present than when more distal landmarks are present. These effects could be produced by either (or both) of two aspects of goal-landmark distance, the absolute distance between the goal and the individual landmarks or relative distances between the landmarks of the array and the goal.

Absolute goal-landmark distance may affect search because of Weber's law, which states that the uncertainty of a measurement is proportional to the magnitude of the property being measured (Barlow, 1982). In other words, the amount of change needed to produce a just noticeable difference in the perception of a stimulus is proportional to the magnitude of the stimulus. A greater amount of change is needed to produce a perceptible difference in the measurement of a stimulus of large magnitude, producing greater uncertainty in measurement of that stimulus. Cheng (1992) applied Weber's law to the spatial domain and found that error in estimating distance and direction to a landmark also increases in proportion to the magnitude of the goal-landmark distance that must be measured. So, in the spatial domain, search may be more accurate with closer landmarks simply because of greater certainty in measuring the distance and direction of a goal from those landmarks. As one moves around a goal location the distance and direction from a close landmark changes much faster than from a distant landmark, making it easier to discern deviations from the correct location (Cheng \& Spetch, 1998). Thus even if all landmarks in an array are weighted equally, more accurate search may be found when only the closest landmark is present than when only a more distant landmark is present.

The relative proximity of landmarks in an array may also influence differential encoding of landmarks. Overshadowing predicts the most salient stimulus in an array will control responding and reduce learning about other stimuli when all are conditioned simultaneously, as a compound (Pavlov, 1927). This is because the stronger stimulus is easier to condition than the overshadowed stimulus, not because conditioning to the overshadowed stimulus is impossible (Kamin, 1969). The concept of overshadowing has been applied to spatial learning in two distinct ways. In some experiments, the modality by which locations were defined was manipulated (SanchezMoreno, Rodrigo, Chamizo, \& Mackintosh, 1999). But in most studies the distance between goal and landmark has been the defining variable (e.g., Gould-Beierle \& Kamil, 1999; Spetch, 1995), and we focus on those studies.

For example, reciprocal overshadowing has been found between intramaze and extramaze cues in radial mazes (for a review, see Chamizo, 2003). Gould-Beierle and Kamil (1999) found overshadowing between different, although entirely visual, cues in an open room environment. They found that landmarks placed on the floor of an experimental room overshadowed the more distant cues, which included the geometry of the room (posters, lights, door, etc.), only when the landmarks on the floor of the room were very close to the goal.

Other studies have allowed for the examination of overshadowing between arrays of similar more proximate cues. Pigeons trained to arrays of landmarks in both an open room and a touchscreen paradigm are more likely to follow a shift of 
the closer landmark (Cheng, 1989) and are more accurate with that landmark when presented alone (Spetch \& Wilkie, 1994). In an experiment designed specifically to test for the effects of overshadowing, Spetch (1995) found that the control exerted over search by a critical landmark depended on that landmark's proximity to the goal relative to other available landmarks, a key prediction of overshadowing. However, these experiments used landmarks that were very close to the goal location, and the results may not generalize to the use of more distant landmarks.

The purpose of the current experiment was to assess the importance of absolute and relative landmark distances when nutcrackers search for a goal, especially when longer goallandmark distances are involved. At greater distances, the closest landmark within an array may not be sufficiently salient to result in overshadowing of other landmarks. We used a design in which we trained three groups of birds to overlapping landmark arrays that included landmarks that were increasingly distant from the goal. In this way, error in tests with a landmark the same distance away from the goal can be compared across groups for which only the relative position of that landmark varies across groups (Spetch, 1995). This design allows differentiation of the effects of absolute from those of relative goal-landmark distances in the encoding of spatial cues by the Clark's nutcracker in an open room environment over a greater range of goal-landmark distances.

\section{Method}

\section{Subjects}

A total of 15 nutcrackers (Nucifraga columbiana) were used in this experiment, 13 experimentally naive nutcrackers and 2 nutcrackers that had previously participated in unrelated operant experiments. During the experiment, the birds were kept at approximately $90 \%$ of their freefeeding weight by controlled daily feeding of a diet of turkey starter, sunflower seeds, parrot pellets, mealworms, pine seeds, and a vitamin supplement. The birds were housed individually with unlimited access to grit and water in a room maintained at $22{ }^{\circ} \mathrm{C}$ with a $14: 10-\mathrm{hr}$ light-dark cycle.

\section{Testing Environment}

The experiment was conducted in a $4.4 \times 2.7 \mathrm{~m}$ room. The birds' entrance to the room, a porthole with a sliding door, was located on the east wall just below a smoked glass observation window with a drawn curtain. The observer entrance door was also on the east wall, north of the porthole. A holding cage where the birds were kept between trials and from which they were released into the room was located at the porthole, outside the experimental room. A Panasonic WV-BL200 video camera was mounted in the ceiling above the test area and connected to a Sony GV-D300 NTSC digital videocassette recorder and Panasonic TR930 video monitor located in the holding area. Birds were observed through the observation window and on the video monitor. The test area was a wooden floor raised $7 \mathrm{~cm}$ above the concrete floor. It began $100 \mathrm{~cm}$ from the east door, extended the entire width of the room, and was covered with approximately 1-2 cm of cellulose substrate. Four centrally located fluorescent lights illuminated the room. Four $42 \times 2.7 \mathrm{~cm}$ (height and outside diameter, respectively) PVC pipes of varying colors and patterns were used as landmarks.

\section{Familiarization}

During this stage the birds became familiar with the experimental room while being trained to enter the room, locate two pine seeds, and then leave the room. There were two trials per day. At the start of each trial, the bird was placed in the holding cage by the porthole. Because nutcrackers prefer lighted areas, the holding room lights were turned off and the experimental room lights turned on and the sliding door between the two rooms was opened. The bird was then allowed to fly into the experimental room, where two shelled pine nuts were sitting exposed at a randomly chosen position in the room on a dark gray 35-mm film canister lid. Once the bird had found and consumed the seeds, the lights were turned off in the experimental room and turned on in the holding room, and the sliding door opened, allowing the bird to return to the holding cage. Familiarization trials lasted for a maximum of $10 \mathrm{~min}$. After the 1st day, substrate was put into the lid to partially cover the seeds. This was done so that the birds used the lid as a cue to the location of the seed. The birds were considered to be familiarized when they readily entered the room, ate the seeds, and left the room. Once the familiarization phase was completed, training began.

\section{Training}

The purpose of this stage was to train the birds to find buried seeds at the goal location defined by a landmark array. The birds were randomly assigned to three groups, with the restriction that the 2 experienced birds were in different groups, and each group was trained with a different landmark array. All birds were given four training trials per day, five days per week. Each group was trained with an array of four landmarks that defined the position of the goal: two shelled pine seeds on the plastic lid. For each group the goal was located at the intersection of the two hypothetical lines connecting the landmarks that were opposite each other in the array. The groups differed in the distance between the goal location and the landmarks. For group close, the distances of the landmarks from the goal were 30, 50, 70, and 90 $\mathrm{cm}$; for group medium, 50, 70, 90, and $110 \mathrm{~cm}$; and for group far, 70, 90, 110, and $130 \mathrm{~cm}$ (as shown in Figure 1). Each landmark in the array was painted a unique color or pattern. They were red, yellow, blue with red stripes, and black with white stripes (closest to furthest landmark, respectively). The colors of the landmarks in each group were as follows: group close, $30 \mathrm{~cm}$ red, $50 \mathrm{~cm}$ yellow, $70 \mathrm{~cm}$ blue with red stripes, $90 \mathrm{~cm}$ black with white stripes; group medium, $50 \mathrm{~cm}$ yellow, $70 \mathrm{~cm}$ blue with red stripes, $90 \mathrm{~cm}$ black with white stripes, $110 \mathrm{~cm}$ red; group far, $70 \mathrm{~cm}$ blue with red stripes, $90 \mathrm{~cm}$ black with white stripes, $110 \mathrm{~cm}$ red, $130 \mathrm{~cm}$ yellow. To ensure the landmark array was the only factor controlling search location, the array was presented in different locations within the experimental arena across trials. However, the goal-landmark array maintained the same orientation relative to the walls and other directional references within the room. These locations were defined by the location of the goal for each individual trial. There were 20 goal locations positioned at least $10 \mathrm{~cm}$ apart. All groups received the array in the same location on each trial. Positions for each trial were selected randomly out of the 20 possible positions without replacement.

Training trials were designed so that the birds would learn to search accurately for buried seeds using the landmark array. Training was divided into four stages. During the first stage of training, the goal was on the surface for the first three trials each day. For the fourth trial, the goal was buried beneath the substrate and the birds were allowed to search until they found the goal, they made 25 digs, or 10 min elapsed, whichever occurred first. During the first 15 days, the goal was gradually buried on the first three trials until just a small portion of the goal (plastic lid) was visible on the surface. After 25 days, the birds were consistently digging close to the goal location and finding the seeds on the fourth trial with the goal completely buried; therefore, we began Stage 2 of training.

In the second stage, which lasted 24 days, the goal continued to be partially buried for the first two trials of the day and was completely buried for the last two trials. During the third stage of training, which continued for 27 days, the goal was buried beneath the surface for all four trials each day. In the fourth stage of training, lasting 5 days, the birds were acclimated to occasional trials without reward. On one randomly chosen trial each day, but never the first trial, neither the seeds nor lid were present in the room, and the trial continued until the birds had dug 10 times. 

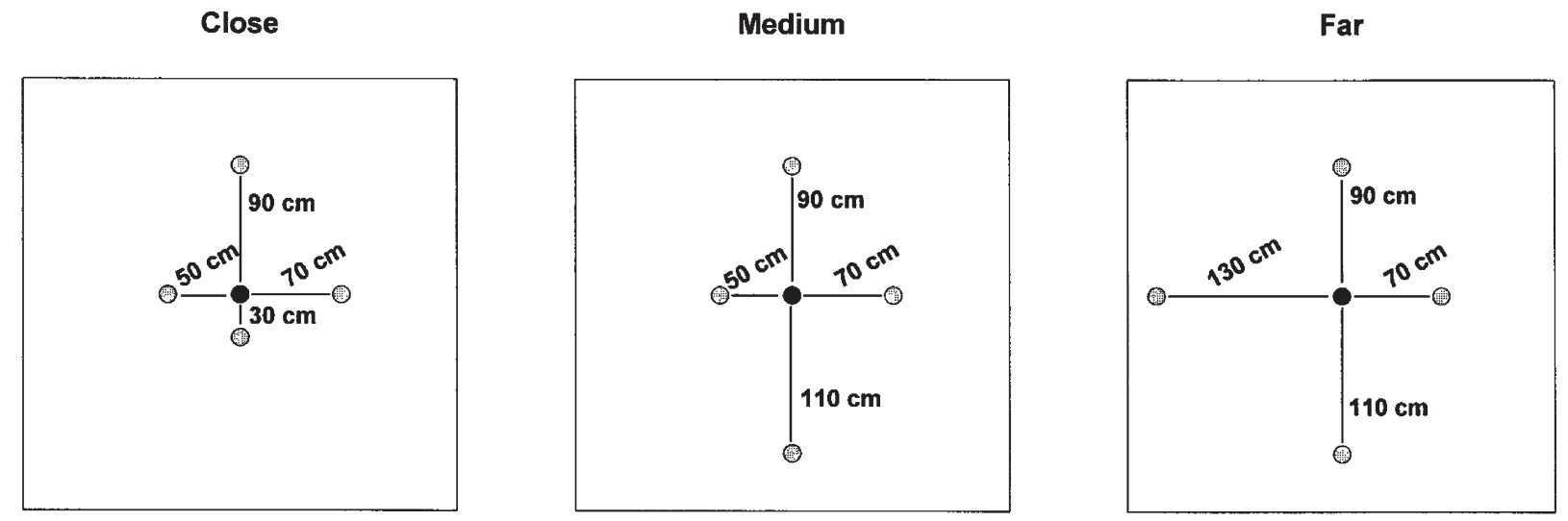

Figure 1. Diagram of landmark array for all three experimental groups. Gray circles represent landmarks; black circles represent the goal location. Goal-landmark distances are labeled for each landmark. Lines are not drawn to scale

Testing

During testing, each bird was given four trials per day, three baseline trials with seeds and one probe trial with no seeds in random order, with the exception that the first trial was always a baseline trial. During baseline trials, all four landmarks were present with the goal buried in the position defined by the array. Testing trials consisted of either a control test or a single landmark test. During control tests, all four landmarks were present without the goal. In single landmark tests, three of the four landmarks were removed. Over 5 days, each individual landmark and the control condition was presented once, using a randomized block design. During baseline trials, the bird was allowed to dig either until it found the seed or for 25 digs, whichever came first. The experimental trials were nonreinforced, thus the bird was allowed to dig 10 times, as introduced in the acclimation stage.

Analysis

All trials were monitored over closed circuit television and videotaped. The videotape was played back on a digital VCR that allowed frame-by- frame playback. Individual bitmap images were made of the landmark array as well as the bird's first five digs (as defined by their beak touching the substrate) for all buried training trials and all testing trials using Adobe Premiere or DV500 Plus (Pinnacle Systems, Braunschweig, Germany). The positions of the goal, the landmark array, and the first five digs were plotted from the bitmaps on an $x-y$ axis using Sigma Scan Pro (Version 5.0, SPSS, Chicago). This allowed determination of the distance between each dig and the goal location independently for the east-west (EW) and the north-south (NS) axes. We then used the Pythagorean theorem to calculate total search error for each dig. Error was measured as the distance of each of the first five digs in a trial from the goal location and averaged for each trial for each bird.

Repeated measures (general linear modeling) analyses of variance (ANOVAs) were used to analyze the data. Subsequent tests, either Fisher's least significant difference (LSD) test or planned comparisons, were carried out only after significant $F$ ratios. We first analyzed total error before completing more detailed analyses of error in the NS and EW axes. Error was determined using an average of the distance of each bird's first five digs from the goal location in each test trial.

\section{Results}

\section{Training}

The training data were divided into three blocks of 60 buried seed trials each and analyzed with a Group $\times$ Block ANOVA.
Performance improved as training progressed, resulting in a significant decrease in mean total error across blocks, $F(2,24)=$ $33.68, p<.05$ (see Figure 2). Group close performed more accurately throughout training, but the difference between groups only approached significance, $F(2,12)=2.94, p>.05$. The Group $\times$ Block interaction was not significant, $F(4,24)=1.34, p>.05$. Subsequent ANOVAs testing group differences during each block were only significant during Block $3, F(2,12)=3.94, p$ $<.05$. A Fisher's LSD analysis showed group close was significantly more accurate than groups medium or far.

\section{Single Landmark Tests}

In general, search error increased as goal-landmark distance increased during single landmark testing (see Figure 3). Because each group was tested with a unique set of goal-landmark distances, we first performed separate analyses of the effects of goal-landmark distance for each group (summarized in Table 1). For both groups close and medium, there was a significant increase in mean search error as goal-landmark distance increased, as reflected in both an overall significant $F$ ratio and a significant linear trend. The pattern of error producing the linear trend was quite different for groups close and medium (see Figure 3). The birds of group close were significantly more accurate with the closest available landmark $(30 \mathrm{~cm})$ than any other single landmark, according to a Fisher's LSD analysis. In contrast, birds in group medium were significantly more accurate with the two closest landmarks $(50 \mathrm{~cm}$ and $70 \mathrm{~cm}$ ) than with the two more distant landmarks $(90 \mathrm{~cm}$ and $110 \mathrm{~cm})$. Group far, for which the landmarks were presented furthest from the goal, did not show any significant effects of goal-landmark distance during the single landmark tests.

Because the effects of goal-landmark distance appeared to vary across groups, we ranked the landmarks within each group by proximity to the goal so that the value 1 was assigned to the closest landmark, 2 to the next closest, and so forth (e.g., the landmark $50 \mathrm{~cm}$ from the goal was assigned Rank 2 for group close but was assigned Rank 1 for group medium). This ranking was then used to compare the groups in a single Group $\times$ Ranked Distance ANOVA. Error increased significantly with the rank of the landmarks across groups, $F(3,36)=18.78, p<.05$. There was also a significant 


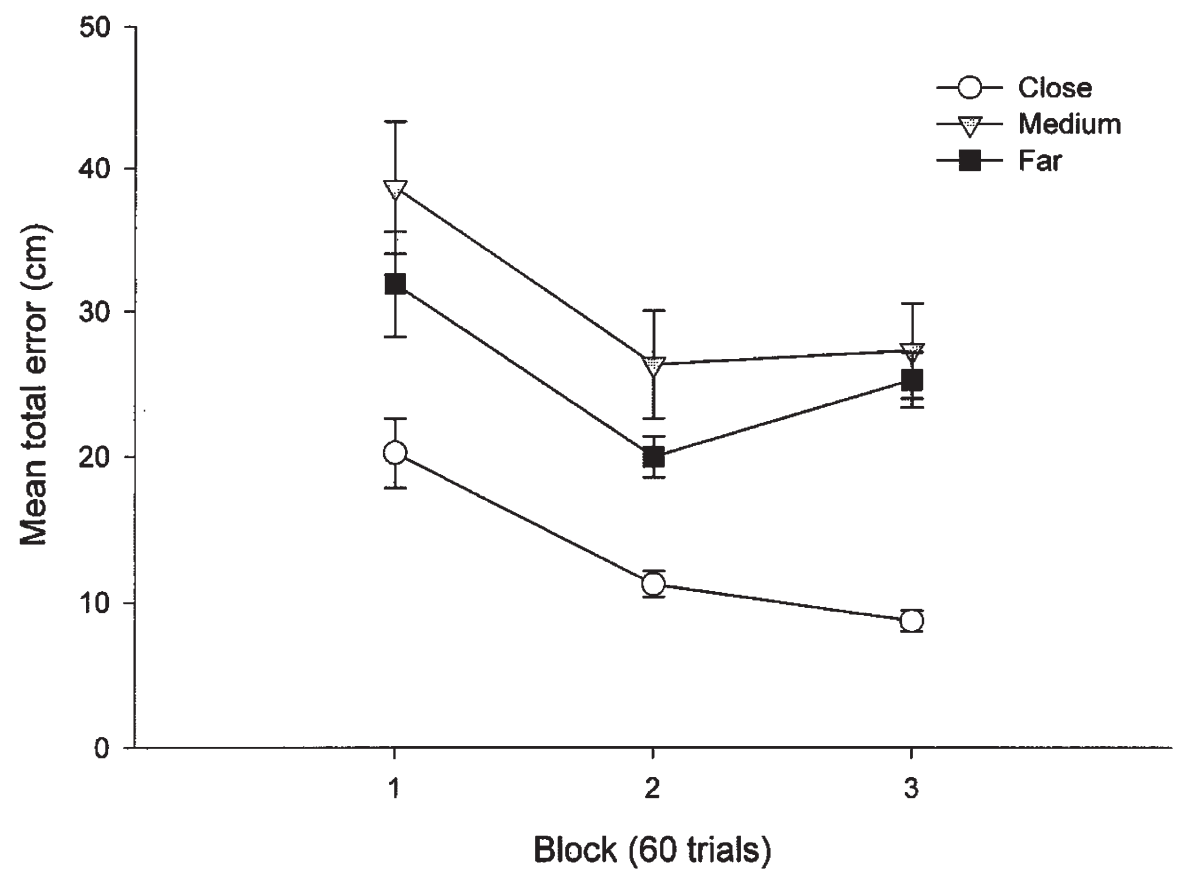

Figure 2. Acquisition error shown by group. Each block represents 60 buried-seed trials. Error bars represent standard error

Group $\times$ Rank Order interaction, $F(6,36)=3.57, p<.05$. There was a significantly greater increase in search error with increasing distance from the goal for groups close and medium than for group far.

\section{Comparison Across Groups}

The landmark arrays used with each of the three groups in this experiment included one landmark $70 \mathrm{~cm}$ from the location of

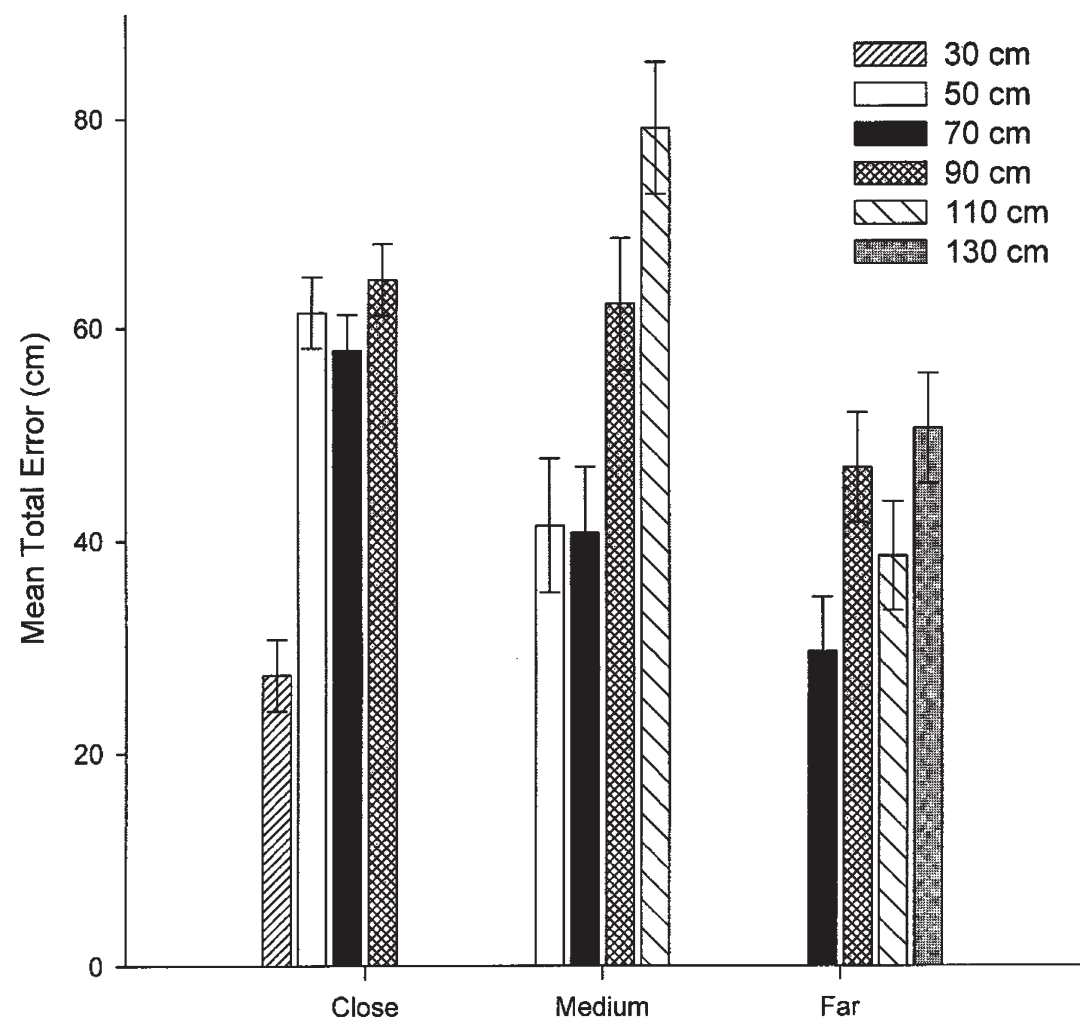

Figure 3. Mean total error in single landmark tests for each group. Error bars represent standard error 
Table 1. Analysis of Variance and Linear Trend Results Comparing Single Landmark Tests for Each Group

\begin{tabular}{lcl}
\hline Group & $F(3,12)$ & $t(12)$ \\
\hline Close & $26.73^{*}$ & $7.27^{*}$ \\
Medium & $8.66^{*}$ & $4.80^{*}$ \\
Far 2.66 & 2.13 & \\
\hline
\end{tabular}

$F$ value is result of analyses of variance comparing error in single landmark tests within groups. Results of linear trend analyses are denoted by $t$ value.

${ }^{*} p<.05$

the goal. An overall analysis of performance by each group when only this landmark was present during single landmark testing was carried out. There was a significant group effect (see Figure 4), $F(2,12)=4.33, p<.05$. Planned contrasts showed that group close exhibited greater error with the $70-\mathrm{cm}$ landmark than group far $(p<.05)$, but there were no significant differences between group medium and either group close or group far (see Figure 5).

There was not a significant group effect, $F(2,12)=1.72, p$ $>.05$, in a similar ANOVA of Performance $\times$ Group with only the $90-\mathrm{cm}$ landmark. The $90-\mathrm{cm}$ landmark was also present in all groups. Unlike the $70-\mathrm{cm}$ landmark, however, it was never the most proximate landmark to the goal within a group.

The $50-\mathrm{cm}$ landmark was closest to the goal in group medium and second closest to the goal in group close. An ANOVA comparing performance of the two groups in $50-\mathrm{cm}$ single landmark tests found that group medium was significantly more accurate when tested with only the $50-\mathrm{cm}$ landmark than was group close, $F(1,8)=7.57, p<.05$.

\section{Discussion}

This study was designed to distinguish between the effects of absolute and relative goal-landmark proximity. The absolute proximity of the $70-\mathrm{cm}$ landmark was held constant while its relative proximity varied across groups. In this way, any differences found between groups during probe trials with only the $70-\mathrm{cm}$ landmark present would be the result of relative landmark proximity. Search accuracy on probe trials increased significantly across groups as the relative proximity of the 70$\mathrm{cm}$ landmark within an array increased. The nutcrackers were more accurate in 70-cm landmark tests when trained with that landmark as the most proximate within an array than when it was one of the less proximate landmarks in the array. The same was also true of tests with the $50-\mathrm{cm}$ landmark. This provides a clear demonstration of overshadowing, with the closest, or most proximate and therefore most salient, landmark within an array controlling search.

Similar qualitative, but statistically nonsignificant, results were found in tests with the 90-cm landmark, which was also present for all three groups. Perhaps this was because the 90$\mathrm{cm}$ landmark was never the closest to the goal for any group. If a fourth group had been added with the $90-\mathrm{cm}$ landmark as the closest landmark, we may have found quantitative difference between the groups. However, the trend toward increased accuracy with the $90-\mathrm{cm}$ landmark in group far (see Figure 3) indicates that overshadowing may not have been as strong in group far. This suggests that the effect of overshadowing may have been even weaker in a fourth group.

Although there were clear effects of overshadowing, these effects decreased as overall goal-landmark proximity increased across groups. This was clearly demonstrated by the significant interaction between group and ranked proximity. If overshadowing on the basis of relative proximity was the sole factor controlling the weighting of the landmarks in the arrays, all three groups should have exhibited the same pattern of error in single landmark tests. Instead, group far showed no decrease in accuracy with decreasing ranked proximity whereas the other two groups did. This and the trend of increasing accuracy with the $90-\mathrm{cm}$ landmark across groups indicates that overshadowing is not the only factor affecting the weighting

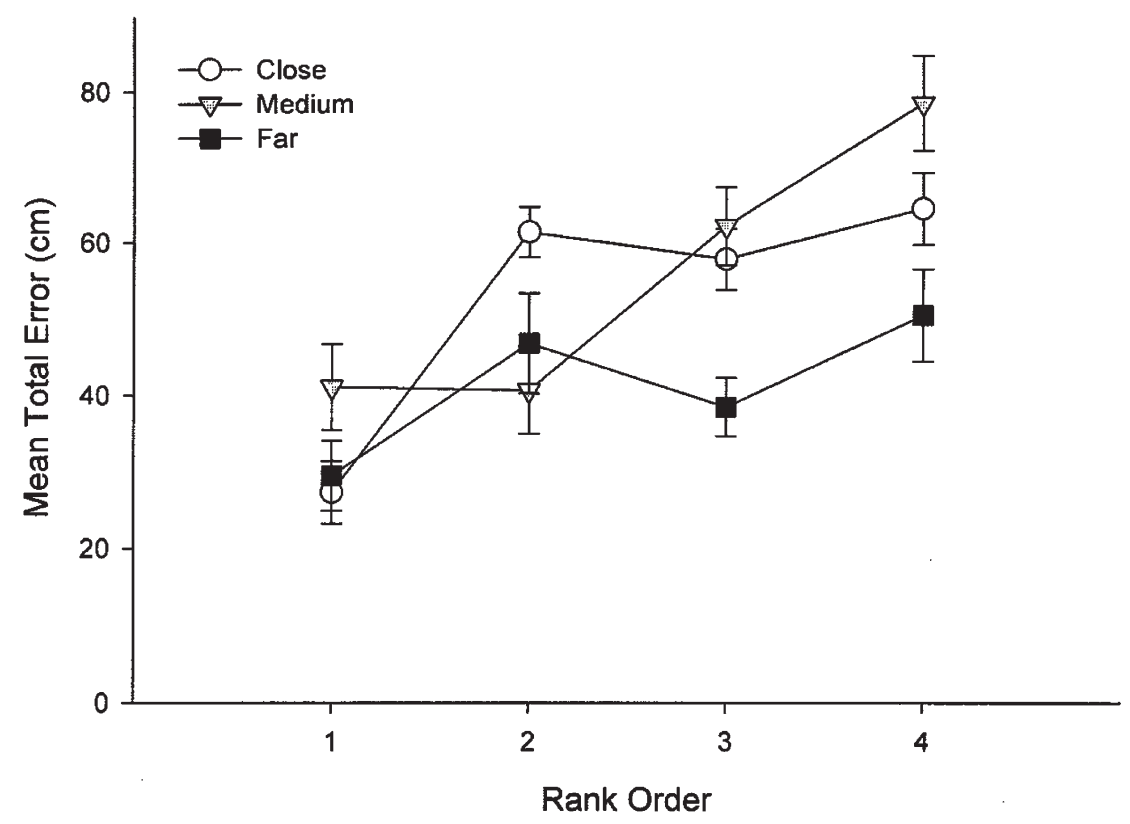

Figure 4. Mean total error for each single landmark test denoted by rank order of those landmarks within a group. Error bars represent standard error 


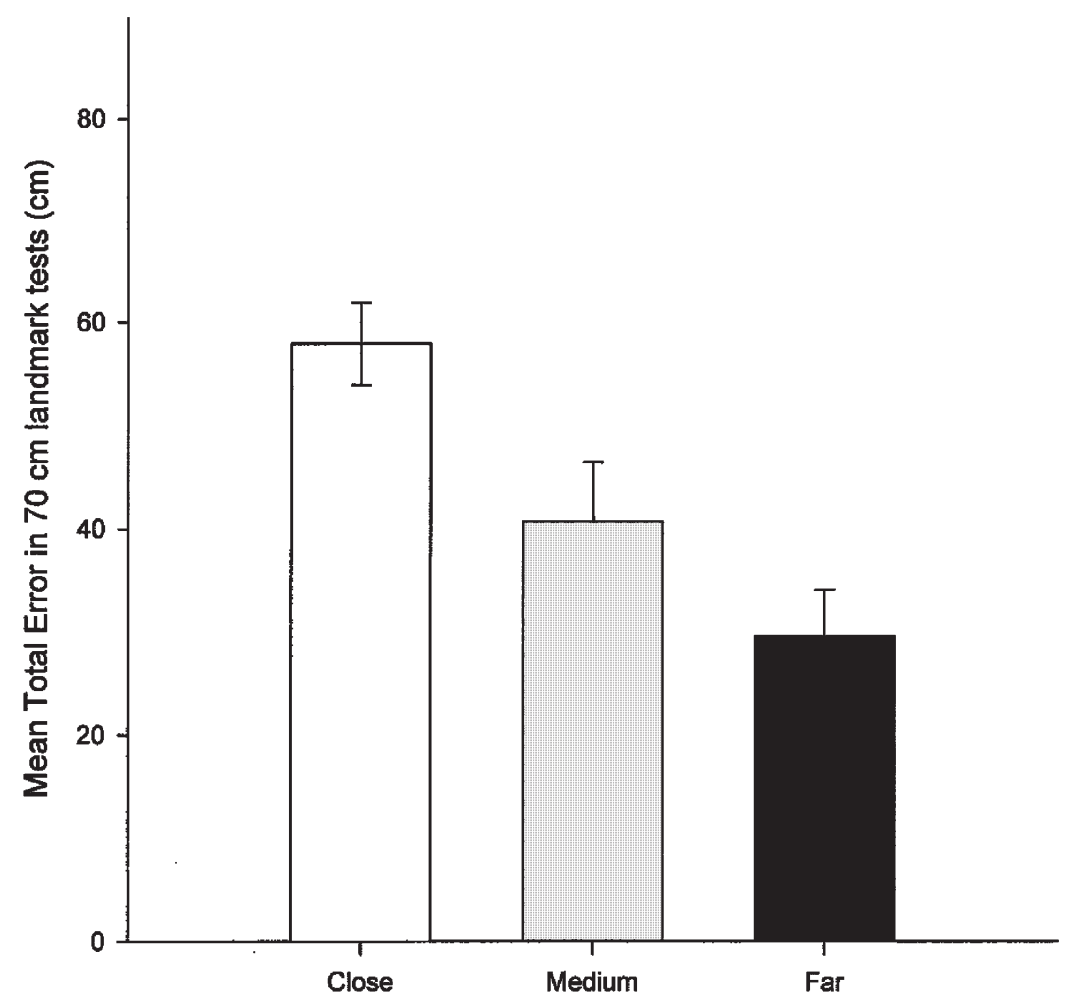

Figure 5. Mean total error in 70-cm single landmark tests for each group. Error bars represent standard error

of landmarks and is probably a minor factor at greater goallandmark distances.

Like group far, group medium also showed smaller effects of overshadowing than group close. The lack of a difference between group medium and any other group in 70-cm landmark tests suggests that the nutcrackers in group medium had learned about more than only the closest landmark in the array. If the most proximate landmark in the array was controlling search in all groups, group far should have been the only group showing any accuracy in tests with only the $70-\mathrm{cm}$ landmark. Instead, the lack of a difference between the error of group medium and group far in 70-cm landmark tests indicates that group medium was not completely ignoring the $70-\mathrm{cm}$ landmark, which was second closest to the goal. This shows that complete overshadowing did not occur in groups medium or far.

It should be noted that the logic of the design used in this experiment differs somewhat from the traditional overshadowing paradigm. In the traditional paradigm, responding to a single conditioned stimulus is compared with responding to the same conditioned stimulus after it has been trained as part of a compound. If that logic were applied to the use of landmarks in spatial learning, the accuracy of a group trained with a single landmark would be compared with accuracy of another group in the same test following training with that landmark in compound with another landmark located closer to the goal. This comparison would be flawed, however. When only a single landmark is present, the only way to encode the goal location is in terms of the distance and direction between the landmark and the goal. With two or more landmarks, there are many more ways to define the goal position in reference to the landmarks. For example, either distance or direc- tion to each of two landmarks could be used (Kamil \& Cheng, 2001). Thus, the design originally developed by Spetch (1995) and used in the current study is more appropriate to the study of overshadowing and goal-landmark distance.

Our experiment is not the first to show a difference in the encoding of the environment with different goal-landmark distances. Gould-Beierle and Kamil (1999) also found that the effects of overshadowing decrease with increasing goal-landmark distance. In their experiment, the close landmarks on the floor of the open room did not overshadow the more distant cues of the perimeter of the room when the close landmarks were further from the goal. Healy and Hurly (1998) found that hummingbirds trained to retrieve food from the middle of an array of flowers used the position of the correct flower in the array in shift tests when the flowers were spaced closely together. However, they used more distant location cues and so did not shift with the array when the flowers were more distantly spaced. This indicates hummingbirds also use more cues as landmark distance increases and may even encode the location of a reward differently as local cues become more distant.

Why does this shift in encoding with increasing goal-landmark distance occur? It is likely due to the increasing difficulty of accurately finding a goal using a single landmark with increasing goal-landmark distance. Error in estimating distance and direction increases in proportion to the magnitude of the goal-landmark distance being measured (Cheng, 1992). To compensate for the increasing error that would result from using only the most proximal landmark in an array when that landmark is far from the goal, the nutcrackers learned about the relationship of increasing numbers of landmarks within the array to the goal. By training nutcrackers to an array of four landmarks ranging from $60 \mathrm{~cm}$ to $105 \mathrm{~cm}$ from 
the goal as well as all 15 possible subsets of those landmarks, Kamil, Goodyear, and Cheng (2001) have demonstrated that nutcrackers are more accurate with more landmarks present when the distance between the goal and each of the landmarks is relatively large.

Encoding multiple landmarks is effective because when the distance between the goal and a landmark is relatively great, nutcrackers tend to be more accurate in determining the direction from the landmark goal than the distance from the landmark goal (Kamil \& Jones 1997, 2000; Kamil et al., 2001). When a single distant landmark is used, direction from that landmark alone is not sufficient to determine a goal location. Therefore, encoding the directional relationships of multiple landmarks to a goal greatly increases accuracy in locating a goal location (Kamil \& Cheng, 2001).

There have been many open field and touch screen studies (Cheng, 1989; Spetch, 1995; Spetch \& Wilkie, 1994) in which animals showed a dependence on close landmarks in a multiple landmark array. Many of these studies have included landmarks at very close distances and found that the subjects relied primarily on a single landmark from the array, as would be expected from our results. Our data also suggest that different results would be found at greater goal-landmark distances. Rather than almost exclusive use of a single landmark from an array, more dependence on multiple landmarks might be seen. There have been studies looking at the use of landmarks equidistant from a goal (Spetch, Cheng, \& MacDonald, 1996; Spetch et al., 1997). None of these, however, measured the degree of dependence on individual landmarks in the array. We suggest that degree of dependence on a particular landmark in this situation would depend on the distance of the landmarks from the goal and whether encoding additional landmarks would be redundant.

In sum, although relative goal-landmark distance is important at relatively short goal-landmark distances, its influence decreases as goal-landmark distance increases. It appears that landmarks at distances further from the goal are not sufficiently salient to overshadow learning about other available landmarks, showing the importance of absolute goal-landmark distance. Because of the effects of Weber's law, it is difficult to accurately locate the goal with a single landmark, or in associative terminology, the closest landmark is no longer salient enough to support strong conditioning. Therefore, instead of encoding only the relationship of the closest landmark to the goal, the nutcracker learns about several landmarks in order to accurately locate the goal. Whether this is done through relative or absolute encoding of landmarks needs to be explored.

\section{References}

Barlow, H. B. (1982). General principles: The senses considered as physical instruments. In H. B. Barlow \& J. D. Mollon (Eds.), The senses (pp. 1-33). Cambridge, England: Cambridge University Press.

Basil, J. A. (1993). Neuroanatomical and behavioral correlates of spatial memory in Clark's nutcrackers. Unpublished doctoral dissertation, University of Massachusetts, Amherst.

Bennett, A. T. D. (1993). Spatial memory in a food storing corvid. 1. Near tall landmarks are primarily used. Journal of Comparative Physiology: Sensory Neural and Behavioral Physiology, 173A193-207.
Chamizo, V. D. (2003). Acquisition of knowledge about spatial location: Assessing the generality of the mechanism of learning. Quarterly Journal of Experimental Psychology: Comparative and Physiological Psychology, 56B, 102-113.

Cheng, K. (1989). The vector sum model of pigeon landmark use. Journal of Experimental Psychology: Animal Behavior Processes, 15, 366-375.

Cheng, K. (1992). Three psychophysical principles in the processing of spatial and temporal information. In W. K. Honig \& J. G. Fetterman (Eds.), Cognitive aspects of stimulus control (pp. 69-88). Hillsdale, NJ: Erlbaum.

Cheng, K., Collett, T. S., Pickhard, A., \& Wehner, R. (1987). The use of visual landmarks by honeybees: Bees weigh landmarks according to their distance from the goal. Journal of Comparative Physiology, $159,469-475$.

Cheng, K., \& Spetch, M. L. (1998). Mechanisms of landmark use in mammals and birds. In S. Healy (ed.), Spatial representation in animals (pp. 1-52). Oxford, England: Oxford University Press.

Collett, T. S., Cartwright, B. A., \& Smith, B. A. (1986). Landmark learning and visuospatial memories in gerbils. Journal of Comparative Physiology: Sensory Neural and Behavioral Physiology, 158A, 835-851.

Gould-Beierle, K. L., \& Kamil, A. C. (1999). The effect of proximity on landmark use in Clark's nutcrackers. Animal Behaviour, 58, $477-488$.

Healy, S., \& Hurly, T. A. (1998). Rufous hummingbirds' (Selasphorus rufus) memory for flowers: Patterns or actual spatial locations?Journal of Experimental Psychology: Animal Behavior Processes, 24, 396-404.

Kamil, A. C., \& Cheng, K. (2001). Way-finding and landmarks: The multiple-bearings hypothesis. Journal of Experimental Biology, 204, 103-113.

Kamil, A. C., Goodyear, A. J., \& Cheng, K. (2001). The use of landmarks by Clark's nutcrackers: First tests of a new model. Journal of Navigation, 54, 429-435.

Kamil, A. C., \& Jones, J. E. (1997, November20). The seed-storing corvid Clark's nutcracker learns geometric relationships among landmarks. Nature, 390, 276-279.

Kamil, A. C., \& Jones, J. E. (2000). Geometric rule learning by Clark's nutcrackers (Nucifraga columbiana)Journal of Experimental Psychology: Animal Behavior Processes, 26, 439-453.

Kamin, L. J. (1969). Selective association and conditioning. In N. J. Mackintosh \& W. K. Honig (Eds.), Fundamental issues in associative learning (pp. 42-64). Halifax, Nova Scotia, Canada: Dalhousie University Press.

Pavlov, I. P. (1927). Conditioned reflexes. (G. V. Anrep, trans.). London: Oxford University Press.

Sanchez-Moreno, J., Rodrigo, T., Chamizo, V. D., \& Mackintosh, N. J. (1999). Overshadowing in the spatial domain. Animal Learning and Behavior, 27, 391-398.

Spetch, M. L. (1995). Overshadowing in landmark learning: Touchscreen studies with pigeons and humans. Journal of Experimental Psychology: Animal Behavior Processes, 21, 166-181.

Spetch, M. L., Cheng, K., \& MacDonald, S. E. (1996). Learning the configuration of a landmark array: I. Touch-screen studies with pigeons and humans. Journal of Comparative Psychology, 110, 55-68.

Spetch, M. L., Cheng, K., MacDonald, S. E., Linkenhoker, B., Kelly, D., \& Doerkson, S. (1997). Use of landmark configuration by pigeons and humans: II. Generality across search tasks. Journal of Comparative Psychology, 111, 14-24.

Spetch, M. L., \& Wilkie, D. M. (1994). Pigeons' use of landmarks presented in digitized images. Learning and Motivation, 25, 245-275.

Submitted July 7, 2003 revised December 2, 2003; accepted December 5, 2003. 